Chimia $45(1991) 228-232$

(c) Schw'eiz. Chemiker-Verband; ISSN 0009-4293

\section{Verhalten von Kupfer, Zink und Cadmium in einem stark belasteten Kalkboden}

\author{
Peter Federer und Hans Sticher*
}

\begin{abstract}
Near a brass foundry, the soil is severely polluted by the heavy metals cadmium, copper, and zinc which have been emitted for more than 80 years. Lime and clay present in the soil result in high $\mathrm{pH}$ and high adsorption capacity, leading to a small mobility of the metals. Therefore, the heavy-metal pollution is localized in the upper $25 \mathrm{~cm}$ of the soil. The enrichment of the metals in the topsoil substantially decreases biological decomposition rate and breakdown of the litter. The fertility of the soil is disturbed and the meagre vegetation on the site is strongly polluted.

The three metals differ in their binding forms and their mobility. Cadmium is particularly adsorbed in the diffuse layer of the cation exchanger or associated with carbonates, which results in a relatively high plant availability. On the other hand, the exchangeable amounts of copper and zinc are small. Main binding forms of these metals are association with carbonates or iron oxides and especially in the case of copper the complexation by humic substances. In the soil solution, hydrated ions are the main species of cadmium and zinc, whereas copper is almost exclusively complexed by dissolved organic matter (DOC). As hydrated ions are preferentially taken up by plants, the availability of cadmium and zinc is greater than that of copper.
\end{abstract}

\section{Einleitung}

Jeder Boden enthält, entsprechend seinem Ausgangsgestein und seinen chemischphysikalischen Eigenschaften einen bestimmten Grundgehalt an Schwermetallen. Einige dieser Elemente sind für den pflanzlichen und tierischen Organismus in kleinen Mengen lebensnotwendig (Spurenelemente, z.B. Co, $\mathrm{Cu}, \mathrm{Fe}, \mathrm{Mn}, \mathrm{Zn}$ ). Eine zu geringe Verfügbarkeit im Boden führt zu Mangelerscheinungen. Andrerseits kann eine zu hohe Konzentration Wachstumsstörungen und Toxizitätssymptome hervorrufen. Von einigen der Schwermetalle ist keine physiologische Notwendigkeit bekannt, sie wirken bereits in sehr kleinen Mengen toxisch und stellen deshalb besonders gefährliche Umweltgifte dar (Cd, $\mathrm{Hg}, \mathrm{Pb}, \mathrm{Tl})$.

Wegen der globalen Verfrachtung von Industrie- und Verkehrsemissionen gelangen heute mit den Niederschlägen oder mit dem atmosphärischen Staub nahezu überall zusätzliche Schwermetallfrachten auf den Boden. Auf landwirtschaftlich genutzten Flächen kommen durch die Düngung, vor allem mit dem Klärschlamm, weitere Men-

\footnotetext{
"Korrespondenz: Prof. H. Sticher Institut für Terrestrische Oekologie ETH Zürich

$\mathrm{CH}-8092$ Zürich
}

gen dazu. Da die Emissionen in der Mehrzahl Punktquellen entstammen (Industriekomplexe, Kehrichtverbrennungsanlagen, Verkehrsknotenpunkte), finden sich in deren Nähe im Boden zuweilen Anreicherungen, welche den natürlichen Zustand um ein Vielfaches übersteigen [1][2]. Diese regionalen Belastungen übertreffen die grossräumigen Schwermetallimmissionen deutlich.

Eine Sanierung hochbelasteter Flächen ist mit einem vertretbaren Aufwand nicht möglich. Falls sich als Sofortmassnahme nicht eine Abschürfung und Deponierung des kontaminierten Bodenmaterials aufdrängt, wird in der Regel eine Umnutzung oder Nutzungsauflassung des betroffenen Areals empfohlen, allerdings nur unter der Voraussetzung, dass keine Gefährdung des Grundwassers besteht.

Die Mobilität der Schwermetalle im Boden, welche sowohl die Pflanzenverfügbarkeit als auch die Verlagerung in den Untergrund bestimmt, wird durch eine Reihe von bodenseitigen Faktoren beeinflusst, vor allem durch den $\mathrm{pH}$, den Humus-Gehalt und den Ton-Gehalt [3]. Die Konzentration im Bodenwasser wird im sauren $\mathrm{pH}-\mathrm{Be}$ reich, der in der überwiegenden Mehrzahl der Böden vorherrscht, durch Adsorptionsprozesse bestimmt. Fällungsreaktionen wie z.B. die Bildung von Carbonaten oder Hydroxiden, spielen daneben nur eine untergeordnete Rolle [4]. Die Verlagerung der Metalle erfolgt vorwiegend in gelöster oder komplexierter Form, doch weisen neuere Untersuchungen auf die Bedeutung von kolloidalen Partikeln als Träger hin [5][6].

$\mathrm{Im}$ vorliegenden Beitrag wird anhand eines stark kontaminierten kalkhaltigen Bodens gezeigt, wie durch chemische Analysen des Sickerwassers, aus Rückschlüssen von Gehaltsbestimmungen in der Bodenmatrix und durch Desorptionsexperimente die relative Mobilität der drei Metalle $\mathrm{Cd}, \mathrm{Cu}$ und $\mathrm{Zn}$ ermittelt werden kann.

\section{Untersuchungsgebiet}

Das Untersuchungsgebiet befindet sich im Bereich eines Buntmetallwerkes, durch dessen Staubemissionen die Böden der Umgebung während Jahrzehnten mit hohen Frachten der Schwermetalle $\mathrm{Cu}, \mathrm{Zn}$ und $\mathrm{Cd}$, vorwiegend in oxidischer Form, belastet wurden. Heute überschreiten die Bodengehalte dieser Elemente im Immissionsgebiet die Richtwerte der Verordnung über Schadstoffe im Boden (VSBo) [7] beträchtlich [8]. Die räumliche Belastungsverteilung zeigt eine deutliche Distanzabhängigkeit zum Emittenten und eine Ausrichtung entsprechend der vorherrschenden Winde. In der Hauptwindrichtung können in landwirtschaftlich oder gartenbaulich genutzten Böden bis zu einer Distanz von $2 \mathrm{~km}$ Richtwertuiberschreitungen festgestellt werden, während gegen den Wind erhöhte Werte nur in unmittelbarer Nähe der Fabrik zu finden sind [8]. Für die vorliegende Untersuchung wurde ein Standort in der am stärksten belasteten Zone nahe des Emittenten ausgewählt. Beim Boden handelt es sich um einen kalkhaltigen Fluvisol, der sich aus alluvialem Feinmaterial über einem Schotterbett entwickelt hat. Die Mächtigkeit der Feinmaterialschüttung schwankt $z$ wischen 50 und $150 \mathrm{~cm}$. Der Versuchsstandort wurde im flachgründigsten Bercich eingerichtet, da dort die Versickerung der Schwermetalle in das Schotterbett und damit ins Grundwasser am ehesten zu befürchten ist. Hauptmerkmale des Bodens sind die Vergesellschaftung von Kalk und Tonmineralien, der daraus resultierende $\mathrm{pH}$ zwischen 7 und 8 und die hohe Kationenaustauschkapazität sowie eine ungehemmte Wasserdurchlässigkeit. Auffällig an den Böden in diesem am stärksten belasteten Gebiet ist eine Auflageschicht aus unzersetzter Gras- und Kräuterstreu, mit einer Dicke von bis zu $3 \mathrm{~cm}$ sowie eine spärliche, lückenhafte Vegetationsdekke.

\section{Material und Methoden}

\subsection{Totalaufschluss und sequentielle Ex-} traktion

Am Versuchsstandort wurde aus einer bis in den Untergrund reichenden Profilgrube schichtweise alle $5 \mathrm{~cm}$ Bodenmaterial für die Laboranalysen und die Modellversuche entnommen. Die Erde wurde bei $40^{\circ}$ ge- 
trocknet, mit einer Backenbrechermühle zerkleinert und mit einem 2-mm-Nylon-Sieb gesiebt. In der gewonnenen Feinerde wurde der Schwermetall-Totalgehalt nach VSBo [7] mit heisser $2 \mathrm{M} \mathrm{HNO}_{3}$ extrahiert und mittels Atomabsorptionsspektroskopie (AAS; Varian Spectra AA 400) bestimmt.Zur Charakterisierung des Bodens wurden der $\mathrm{pH}$ (in $0.01 \mathrm{M} \mathrm{CaCl}_{2}$ ) sowie der Gehalt an organischem Material und Carbonat gemessen.

Zur Erfassung der Bindungsformen von Schwermetallen im Boden schlagen verschiedene Autoren sequentielle Extraktionen vor [9-13]. Dabei wird versucht, durch zunehmende Stärke der Extraktionsmittel die Schwermetalle entsprechend ihren Bindungsformen zu extrahieren. Eine scharfe Trennung und Zuordnung der Bindungsformen ist aber leider nicht möglich. So sind die Ergebnisse der verschiedenen Methoden nicht direkt vergleichbar. Je nach Bodentyp und Fragestellung eignet sich die eine oder andere Methode besser. Für den stark kalkhaltigen Boden des Untersuchungsgebiets erwies sich die folgende sechsteilige Extraktionssequenz [13] am geeignetsten:

\section{Schritt: $\quad$ IM $\mathrm{MgCl}_{2}$}

Mit der $\mathrm{MgCl}_{2}$-Lösung werden die diffus [14] gebundenen und austauschbaren Kationen extrahiert.

\section{Schritt: $25 \% \mathrm{AcOH}$}

Mit der $\mathrm{AcOH}$ werden die Carbonate gelöst und die in der äusseren Koordinationssphäre [14] an der mineralischen Phase gebundenen Kationen extrahiert.

\section{Schritt: $\quad$ Im $\mathrm{NH}_{2} \mathrm{OH}$ in $25 \% \mathrm{AcOH}$}

Durch das reduzierende Extraktionsmittel werden in der inneren Koordinationssphäre [14] an Oxide und Tonmineralien gebundene Kationen freigesetzt.

\section{Schritt: $\quad 30 \% \mathrm{H}_{2} \mathrm{O}_{2}$}

Durch die Oxidation des organischen Materials werden die an die organische Substanz komplex gebundenen Kationen extrahiert.

\section{Schritt: $\quad 0.5 \mathrm{~m} \mathrm{HCl}$ \\ 6. Schritt: $\quad 4 \mathrm{M} \mathrm{HNO}_{3}$}

Durch diese starken Säuren werden die Kationen aus dem Kristallgitter von Eisenund Aluminiumoxiden extrahiert.

Für jede Sequenz wurden $12.5 \mathrm{~g}$ Feinerde in $100 \mathrm{ml}$ PE-Flaschen eingewogen und nacheinander mit je $50 \mathrm{ml}$ Extraktionslösung $24 \mathrm{~h}$ offen geschüttelt. Nach jedem Extraktionsschritt wurde die Lösung abzentrifugiert und über Papierfilter (Schleicher und Schuell $589^{3}$ ) in PE-Flaschen abfiltriert. Zwischen den einzelnen Extraktionsschrit-

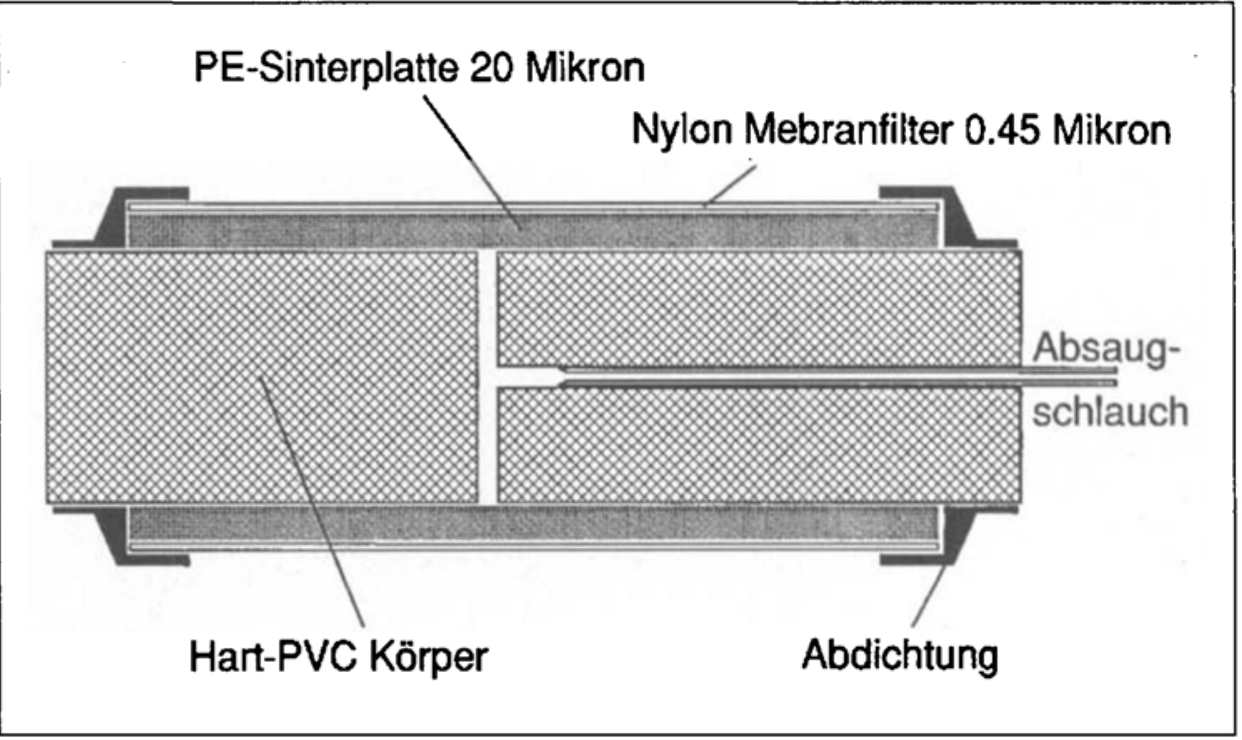

Fig. 1. Aufbau der eingesetzten Nylon/PE-Saugkerzen

ten wurde der Zentrifugenrückstand zweimal mit $\mathrm{H}_{2} \mathrm{O}$ nanopure gewaschen. Die Extrakte 1 bis 4 wurden für die Lagerung und Analyse mit $50 \mathrm{ml}$ konz. $\mathrm{HNO}_{3}$ angesäuert.

\subsection{Sorptionsexperimente}

Für die Erfassung der Kompetition der Schwermetalle um die Bindungsplätze und deren Einfluss auf die SchwermetallDesorption wurden in Batchversuchen je $3 \mathrm{~g}$ Feinerde mit steigenden Konzentrationen von $\mathrm{Zn}$, resp. $\mathrm{Pb}$ versetzt und während $24 \mathrm{~h}$ bei $25^{\circ}$ offen geschüttelt. Als Grundelektrolyt dienten $30 \mathrm{ml} 0.01 \mathrm{M} \mathrm{Ca}\left(\mathrm{NO}_{3}\right)_{2}$ bzw. $\mathrm{Mg}\left(\mathrm{NO}_{3}\right)_{2}$. Nach kurzem Zentrifugieren bei $2000 \mathrm{rpm}$ wurden die Lösungen über $0.45 \mu \mathrm{m}$ Zelluloseacetat-Membranen filtriert und vor der Analyse mit $\mathrm{HNO}_{3}$ angesäuert.

\subsection{Gewinnung von Bodenwasser}

Zur Gewinnung von Bodenwasser für die Analyse von Nährstoff-Gehalten werden üblicherweise Keramik- oder Alumi-
niumoxid-Sinterkerzen eingesetzt. Wegen ihrer hohen Adsorptionskapazität für Kationen eignen sie sich jedoch nur bedingt für die Erfassung von Spurenmengen von Schwermetallen im Sickerwasser [15]. In der vorliegenden Arbeit wurden daher Kunststoff-Kerzen (Fig. I) verwendet, wie sie von Grossmann beschrieben wurden [15]. Bei diesen ist auf einem Hart-PVC-Kern eine $1.5 \mathrm{~mm}$ dicke PE-Sinterplatte (Ligacon $U H M W-P E 1 / 16^{\prime \prime}, 20 \mu$ ) und eine $0.45-\mu \mathrm{m}-$ Filtermembran (PallNXG) aus Nylon mit PEStützgewebe aufgezogen. Die Stirnflächen der Sinterplatte und Filtermembran sind mit einer Silikon-Masse und einem Schrumpfschlauch abgedichtet. Insgesammt wurden je 3 Kerzen in 20 und in $40 \mathrm{~cm}$ Tiefe installiert. Vor dem Einbau wurden die Kerzen nacheinander mit $1 \mathrm{M} \mathrm{HCl}, 1 \mathrm{M} \mathrm{NaOH}$ und mit vollentsalztem $\mathrm{H}_{2} \mathrm{O}$ gespült. Nach dem Einbau wurden die ersten $200 \mathrm{ml}$ der gewonnenen Bodenlösungen verworfen. Die Probengewinnung erfolgt über je $4 \mathrm{~d}$ bei einem Innendruck von 100-300 mbar.

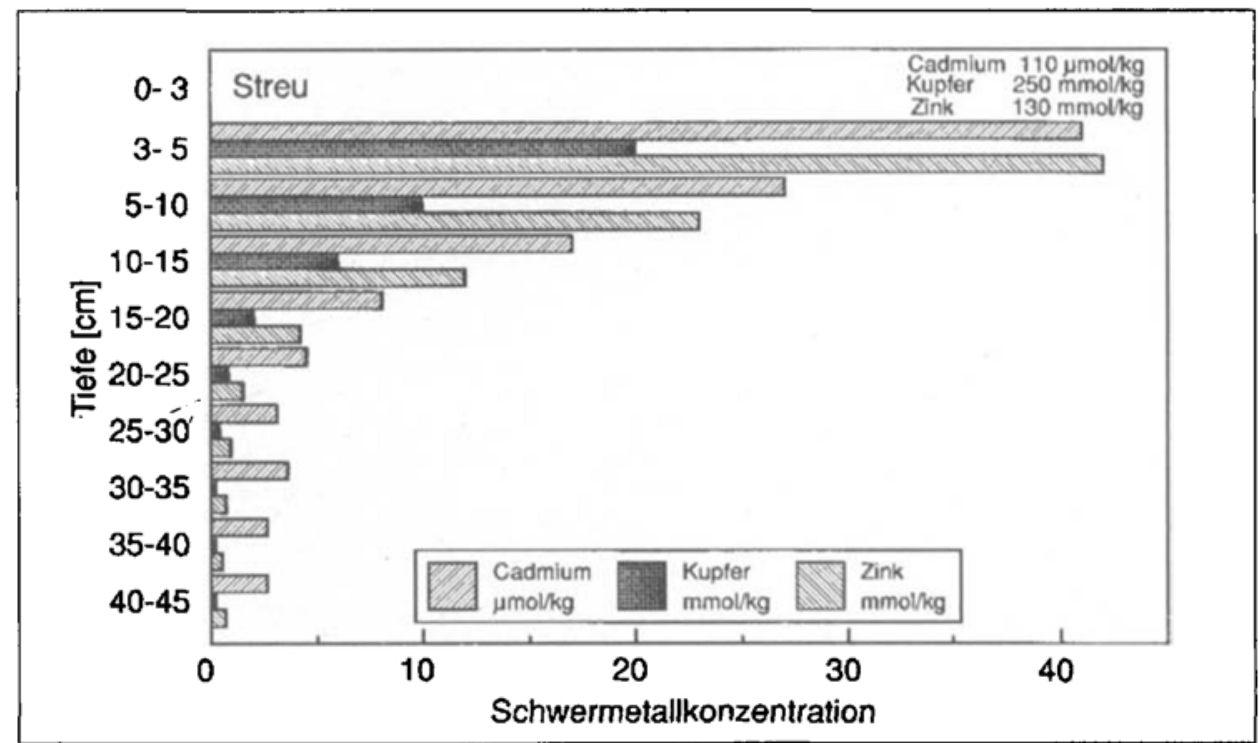

Fig. 2. Konzentrationsprofil der Schwermetalle $C d$. $C u$ und $\mathrm{Z} n$ in einem stark belasteten, kalkhaltigen Fluvisol. Die Richtwerte der VSBo [7] betragen $7.1 \mathrm{mmol} / \mathrm{kg}$ für Cd, $0.79 \mathrm{mmol} / \mathrm{kg}$ für Cu und $3.1 \mathrm{mmol} / \mathrm{kg}$ für Zn, hzgl. 20-cm-Bodentiefe. 


\subsection{Analysemethoden}

Die Analyse der Schwermetall-Gehalte in den Extraktions- und Sorptionslösungen erfolgte mit AAS. Für die Analyse des Bodenwassers kamen folgende Methoden zur Anwendung: 1. Cd, $\mathrm{Cu}$ und $\mathrm{Zn}$ mittels Gra-
phitrohr-AAS; 2 . Na, K, Ca und Mg mittels ICP-AES; 3. Chlorid, Nitrat, Sulfat und Phosphat mittels HPLC; 4. Carbonat mittels FIA; 5. 'gelöster organischer Kohlenstoff' mit einem DOC-Analyzer; sowie $\mathrm{pH}$ und Leitfähigkeit potentiometrisch.
Fig. 3. Relative Anreicherung von $C d$ und $C u$ in der Profiltiefe im Vergleich mit $\mathrm{Zn}$
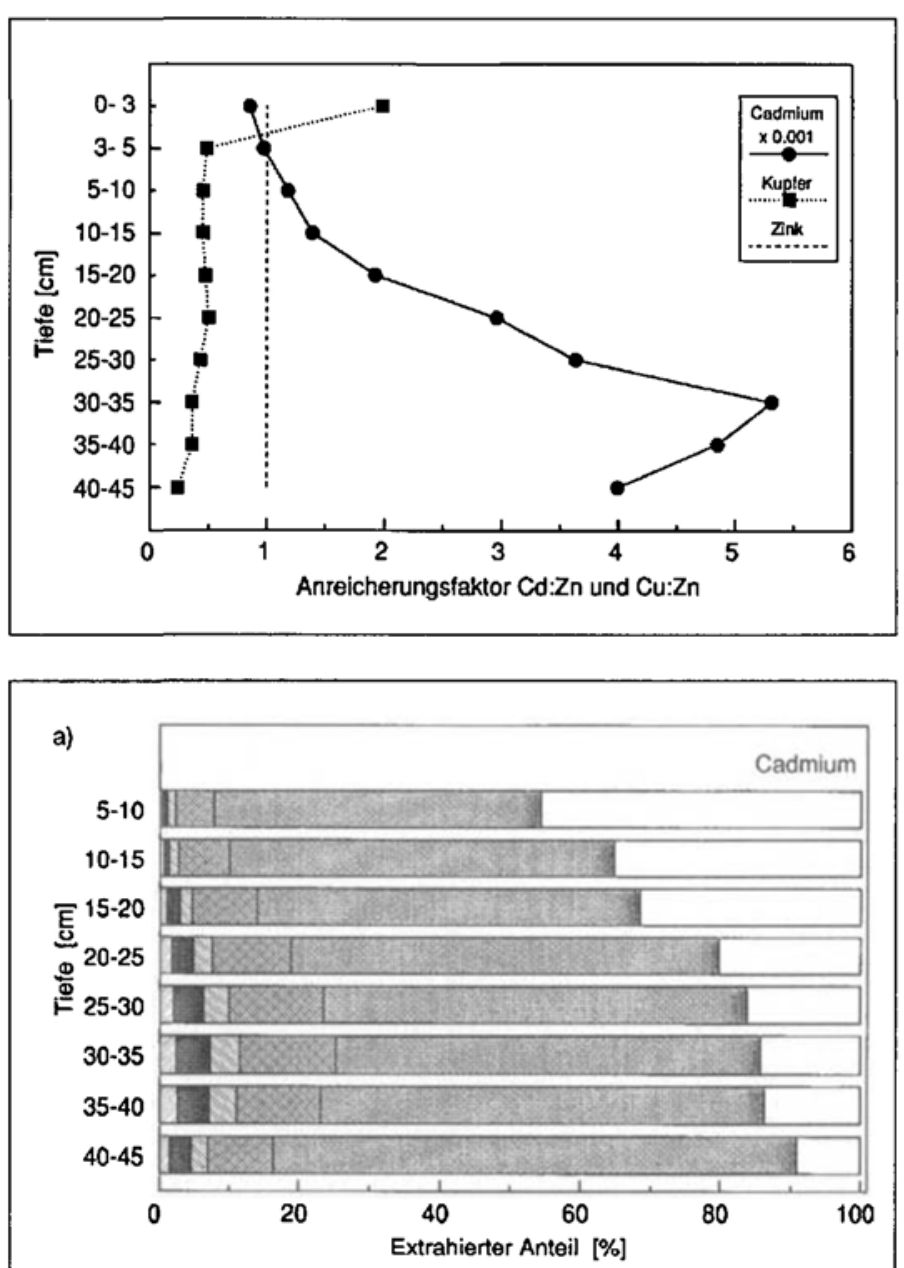

b)
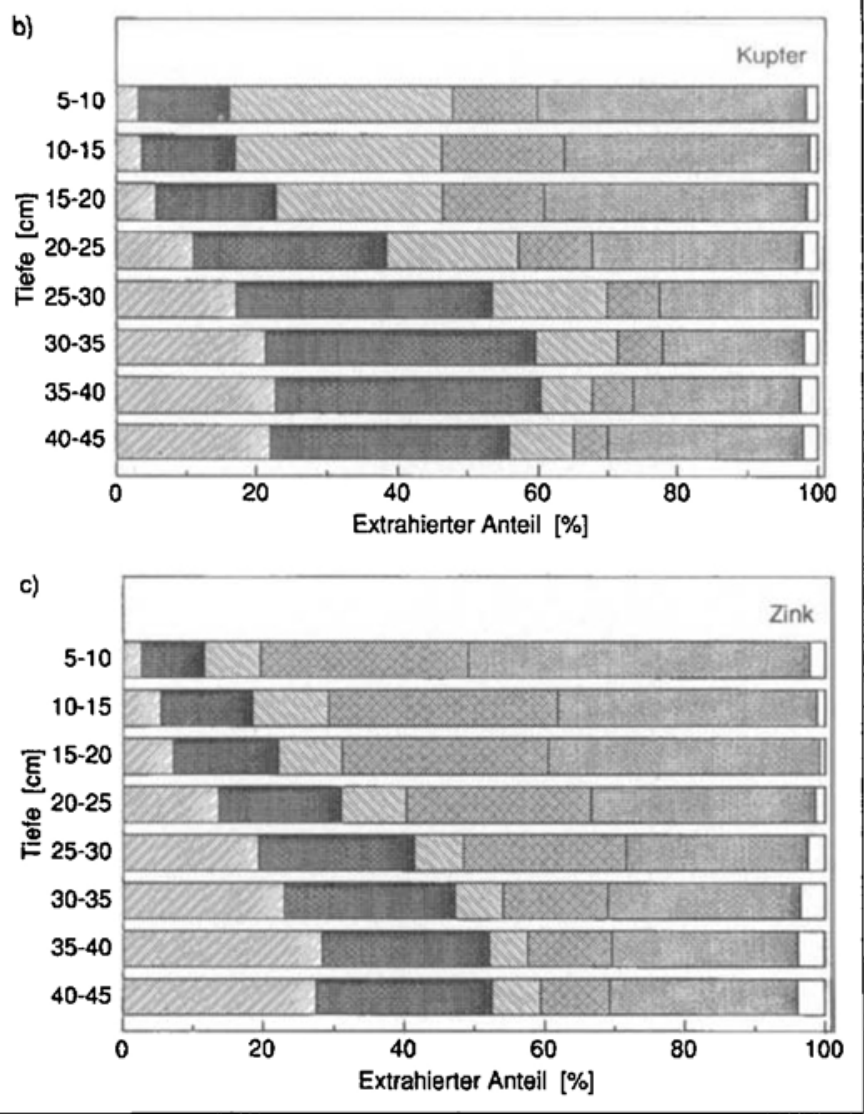

\section{Resultate und Diskussion}

Die Schwermetall-Verteilung im Profil zeigt für $\mathrm{Cd}, \mathrm{Cu}$ und $\mathrm{Zn}$ eine hohe Akkumulation im Oberboden (bis $20 \mathrm{~cm}$ ), mit einer raschen Abnahme in die Tiefe (Fig. 2 und 3). Trotz des jahrzehntelangen Eintrags ist die Belastungsfront bis heute nicht tiefer als $25 \mathrm{~cm}$ vorgedrungen. Die Gehalte im Unterboden $(20-40 \mathrm{~cm})$ sind gegenüber Referenzböden im Schweizer Mittelland [16] nicht erhöht. Gründe für die geringe Mobilität sind der hohe Ton- und Carbonat-Gehalt des Bodens sowie der im alkalischen Bereich liegende $\mathrm{pH}$-Wert [3]. Die übermässige Akkumulation im Oberboden ist charakteristisch für anthropogene Belastungen. Dies wird durch die Ergebnisse von Immissionsmessungen im Untersuchungsgebiet [17] und durch die räumliche Verteilung der Bodenbelastung in Abhängigkeit [8] zur Emissionsquelle bestätigt. Die selektive Anreicherung von einzelnen Elementen durch die natürlichen Bodenbildungsprozesse ist demgegenüber vernachlässigbar.

Auffällig sind die sehr hohen Schwermetall-Konzentrationen in der organischen Auflage. Bei deren Beurteilung muss jedoch das unterschiedliche Raumgewicht und das spezifische Gewicht des Bodenmaterials berücksichtigt werden. Bei einem Raumgewicht von $0.4 \mathrm{~kg} / \mathrm{dm}^{-3}$ in der Streuschicht und 1.2 im obersten mineralischen Horizont $(3-5 \mathrm{~cm})$ enthalten die beiden Schichten pro Volumeneinheit praktisch die selbe Menge $\mathrm{Cd}$ und $\mathrm{Zn}$. Cu dagegen ist auch auf dieser Vergleichsbasis in der Streuschicht noch um den Faktor 4 angereichert. Das Kupferoxid des Sedimentationsstaubes ist wesentlich schlechter wasserlöslich als die Oxide von $\mathrm{Cd}$ und $\mathrm{Zn}$. Es wird deshalb mit dem Niederschlagswasser weniger $\mathrm{Cu}$ in den Boden eingewaschen, was sich in der relativen Anreicherung gegenüber den andern Elementen ausdrückt. Die Bioakkumulation durch die Pflanzen ist dabei zu vernachlässigen, liegen doch die Cu-Gehalte in der Vegetation des Standortes zwischen 0.1 und $1 \mathrm{mmol} /$ kg Trockenmasse [18].

Die für den Standort untypische Streuschicht ist die Folge der hohen Schwermetall-Gehalte. Tyler [19] wies einen negativen Einfluss von hohen Schwermetall-Gehalten auf die Aktivität der Bodenmikroorganismen und den Abbau der organischen Substanz im Boden nach. Ma [20] fand eine markante Reduktion der Fertilität von Regenwürmern und ihrer Streutransportleistung in Cu-belasteten Böden. Auf den Flächen mit der höchsten Kontamination im Untersuchungsgebiet sind tatsächlich keine Regenwürmer mehr zu beobachten. Durch ihr Fehlen findet keine Durchmischung und Einarbeitung der frischen Streu in den Boden statt. Dadurch wird der mikrobielle Abbau zusätzlich erschwert. Die stark schwermetallhaltige Streuschicht hat einen direkten Einfluss auf die Bodenfruchtbarkeit. In den betreffenden Wiesen ist die Verjüngung der Pflanzendecke beeinträchtigt, und die $\mathrm{Gr}$ Fraktionen der sequenticlen Extraktion an den Gehalten von $\mathrm{Cd}, \mathrm{Cu}$ und $\mathrm{Zn}$ (die Summe der sechs Extraktionsschritte entspricht $100 \%$ ) 
Tab. 1. Zusammenstellung der Messwerte in der Bodenlösung während der Messperiode Dez. 90 bis April 91

\begin{tabular}{|c|c|c|c|}
\hline & & $18 \mathrm{~cm}$ Tiefe & $40 \mathrm{~cm}$ Tiefe \\
\hline $\mathrm{pH}$ & & $7.5-8.3$ & $7.6-8.3$ \\
\hline Leitfähigkeit & $\mu \mathrm{S} / \mathrm{cm}$ & $330-650$ & $350-440$ \\
\hline DOC & $\mathrm{mg} / \mathrm{l}$ & $120-220$ & $12-120$ \\
\hline Carbonat & $\mathrm{mm}$ & $2.8-3.9$ & $3.7-4.9$ \\
\hline Phosphat & $\mu \mathrm{M}$ & $\left.<2^{a}\right)$ & $\left.<2^{a}\right)$ \\
\hline Sulfat & $\mu \mathrm{M}$ & $54-730$ & $50-120$ \\
\hline Nitrat & $\mu \mathrm{M}$ & $<75 b)$ & $\left.<20^{\mathrm{b}}\right)$ \\
\hline $\mathrm{Na}$ & $\mu \mathrm{M}$ & $47-87$ & $25-45$ \\
\hline $\mathrm{K}$ & $\mu \mathrm{M}$ & $\left(3.5^{\mathrm{a}}\right)$ & $\left(3.5^{\mathrm{a}}\right)$ \\
\hline $\mathrm{Ca}$ & $\mathrm{mM}$ & $1.4-5.7$ & $7-2.2$ \\
\hline $\mathrm{Mg}$ & $\mu \mathrm{M}$ & $52-210$ & $46-3$ \\
\hline $\mathrm{Cd}$ & $\mathrm{nm}$ & $\left.<1.3^{\circ}\right)$ & $\left.<0.5^{b}\right)$ \\
\hline $\mathrm{Cu}$ & $\mu \mathrm{M}$ & $0.17-1.4$ & $0.08-0.21$ \\
\hline $\mathrm{Zn}$ & $\mu \mathrm{M}$ & $0.05-1.5$ & $0.03-0.15$ \\
\hline
\end{tabular}

a) Phosphat und $\mathrm{K}$ liegen unterhalb der Nachweisgrenze der HPLC respektive des ICP-AES.

b) Nur einzelne Werte liegen über der Nachweisgrenze.

Tab. 2. Mit $0.01 \mathrm{M} \mathrm{Mg}\left(\mathrm{NO}_{3}\right)_{2}$ desorbierte Gehalte an $\mathrm{Cd}$, Cu und $\mathrm{Zn}$ in mmollkg und Promille des Totalgehaltes $\left(\mathrm{pCO}_{2}=0.0003\right.$ bar, $\left.25^{\circ}\right)$

\begin{tabular}{llllll}
\hline & $\begin{array}{l}\text { Tiefe } \\
3-5 \mathrm{~cm}\end{array}$ & $5-10 \mathrm{~cm}$ & $10-15 \mathrm{~cm}$ & $15-20 \mathrm{~cm}$ & $20-25 \mathrm{~cm}$ \\
\hline & $\mu \mathrm{mol} / \mathrm{kg}$ & $\mu \mathrm{mol} / \mathrm{kg}$ & $\mu \mathrm{mol} / \mathrm{kg}$ & $\mu \mathrm{mol} / \mathrm{kg}$ & $\mu \mathrm{mol} / \mathrm{kg}$ \\
$\mathrm{Cd}$ & 0.43 & 0.1 & 0.05 & 0.02 & $<0.18$ \\
$\mathrm{Cu}$ & 210 & 97 & 60 & 28 & 7 \\
$\mathrm{Zn}$ & 205 & 31 & 9.9 & $<3$ & $<3$ \\
& & & & & \\
$\mathrm{Cd}$ & 10.5 & 3.5 & 2.9 & Promille & Promille \\
$\mathrm{Cu}$ & 10.5 & 9.7 & 10 & 2.4 & 8.5 \\
$\mathrm{Zn}$ & 4.9 & 1.4 & & 0.8 & 0.5 \\
\hline
\end{tabular}

asnarbe zeigt deutliche Lücken [18][21]. Lattich, der als Testpflanze auf der Versuchsparzelle gezogen wurde, wies Schwermetall-Gehalte auf (Cd: $4.4 \mathrm{ppm}, \mathrm{Cu}$ : $50 \mathrm{ppm}$ und $\mathrm{Zn}: 860 \mathrm{ppm}$, bzgl. Blattrokkenmasse), welche zum Zeil über den zulässigen Höchstgehalten [22] lagen. Die Wuchsleistung war gegenüber Pflanzen auf einer unkontaminierten Vergleichsfläche um $70 \%$ reduziert $[18][21]$.

Die sequentielle Extraktion (Fig. 4a-c) zeigt, dass nur ein kleiner Prozentsatz von $\mathrm{Cu}$ und $\mathrm{Zn}$ in austauschbarer Form vorliegt. Dagegen ist der Anteil an austauschbarem $\mathrm{Cd}$, besonders im stark kontaminierten Oberboden mit bis zu $45 \%$ auch prozentual bedeutend. Entsprechend kann die Cd-Mobilität durch konkurrenzierende Kationen (in kalkhaltigen Böden vorwiegend $\mathrm{Ca}$ ) erhöht werden. Cd wird dadurch verstärkt verlagert und reichert sich im schwächer belasteten Unterboden gegenüber $\mathrm{Cu}$ und $\mathrm{Zn}$ an (Fig. 3). Diese Verlagerung von Cd erfolgt langsam und, bedingt durch die hohe Adsorptionskapazität des Bodens, nur über kurze Distanzen. Die Cd-Gehalte des natürlichen Bodenwassers (Tab, 1 ) in der Tiefe von 18 und $40 \mathrm{~cm}$ liegen unterhalb der Nachweisgrenze der flammenlosen AAS von $10^{-9} \mathrm{~mol} / \mathrm{l}$. Eine Auswaschung in die Schotterschicht und ins Grundwasser ist deshalb trotz der erhöhten Mobilität im Oberboden unter den heutigen Bedingungen nicht zu befürchten. Die erhöhte Mobilität von $\mathrm{Cd}$ wirkt sich aber auf die Pflanzenverfügbar- keit aus. Im Versuch mit Lattichpflanzen [18] betrug der Transferquotient (Gehalt in der Pflanze geteilt durch Gehalt im Boden)

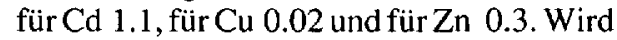
der Wert für Cu gleich 1 gesetzt, berechnet sich für Cd ein relativer Quotient von 55 und für $\mathrm{Zn}$ von 15.

Ein bedeutender Anteil aller drei Schwermetalle liegt in der AcOH-extrahier-

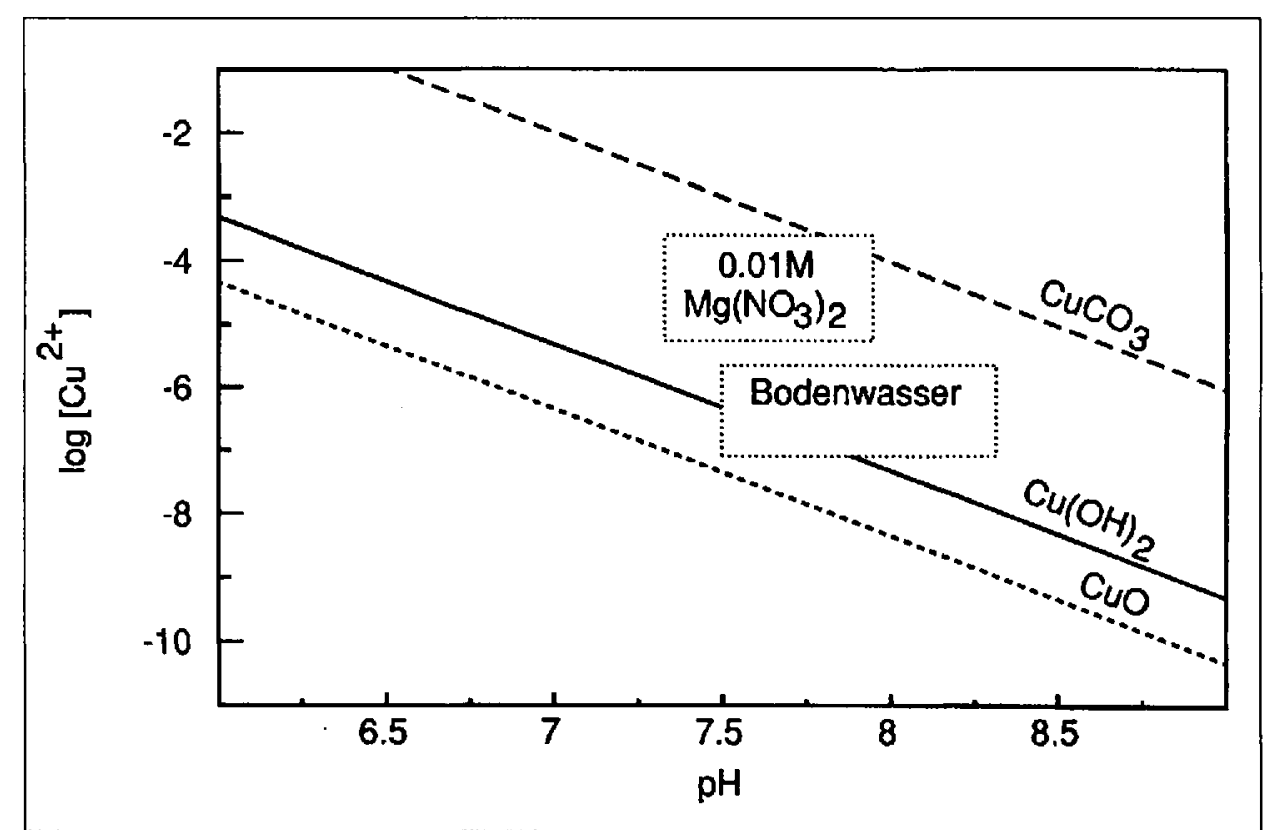

Fig. 5. Löslichkeitsdiagramm ausgewählter Cu-Verbindungen (nach Lindsay [26]) mit den Bereichen der pHWerte und $C u$-Gesamtkonzentrationen in den Desorptionslösungen und im natïrlichen Bodenw'usser $\left(\log p\left(\mathrm{CO}_{2}\right)\right.$ : $-3.52,25^{\circ}$ ) baren Fraktion vor. Längerfristig kann dieser Anteil nur durch sehr hohe Konzentrationen konkurrenzierender Kationen in der Bodenlösung und durch die Auflösung der Carbonate infolge der fortlaufenden Protonenzufuhr aus der Atmosphäre (saurer Regen, C-Kreislauf) mobilisiert werden. Die Löslichkeit von Carbonaten ist vom $\mathrm{CO}_{2}$ Partialdruck abhängig, welcher im Boden saisonal im Bereich von 0.32 mbar (= Atmosphärenluft) bis 50 mbar (bei hoher biologischer Aktivität) schwankt. Entsprechend wird die Freisetzungsrate der in dieser Fraktion gebundenen Kationen in Zeiten hoher biologischer Aktivität beeinflusst. Dies könnte sich vor allem beim Cd auswirken, das zur Hauptsache in dieser Bindungsform vorliegt.

Bei der $\mathrm{H}_{2} \mathrm{O}_{2}$-Extraktion fällt im Vergleich zu $\mathrm{Cd}$ und $\mathrm{Zn}$ der hohe Anteil von $\mathrm{Cu}$ auf, der in dieser Fraktion vorliegt. Der $\mathrm{Cu}$ Anteil korreliert mit dem Humus-Gehalt in den einzelnen Schichten. Im Oberboden liegt bei einem Gehalt von $2.5 \%$ organischem Kohlenstoff bis $30 \%$ von $\mathrm{Cu}$ in dieser Fraktion gebunden vor. Die bevorzugte Bindung von $\mathrm{Cu}$ an die Huminstoffe lässt sich durch die hohe Stabilitätskonstante von Kupferhumat-Komplexen erklären ( $\log K_{2}$ für Cu: 8.9; für Zn 5.53 und für Cd: 5.23 [23]). Cu bildet aber auch stabile Komplexe mit einem Teil der löslichen niedermolekularen Verbindungen (DOC), welche aus der Streu ausgewaschen oder beim Abbau der organischen Substanz durch Mikroorganismen gebildet werden. Durch die Komplex-Bildung in der Lösungsphase wird die Mobilität von $\mathrm{Cu}$ erhöht, so dass sowohl die Desorptionslösungen (Tab. 2) (mit $0.01 \mathrm{M}$ $\mathrm{Ca}\left(\mathrm{NO}_{3}\right)_{2}$ und $\left.\mathrm{Mg}\left(\mathrm{NO}_{3}\right)_{2}\right)$ als auch die natürliche Bodenlösung (Tab. I) in Bezug auf $\mathrm{Cu}(\mathrm{OH})_{2}$ übersättigt (Fig. 5) sind. Berechnungen mit dem Speziierungsprogramm SOILCHEM [24] haben gezeigt, dass in der 
CHIMIA 4.5 (1991) Nr. 7/8 (Juli/Augusl)

a)

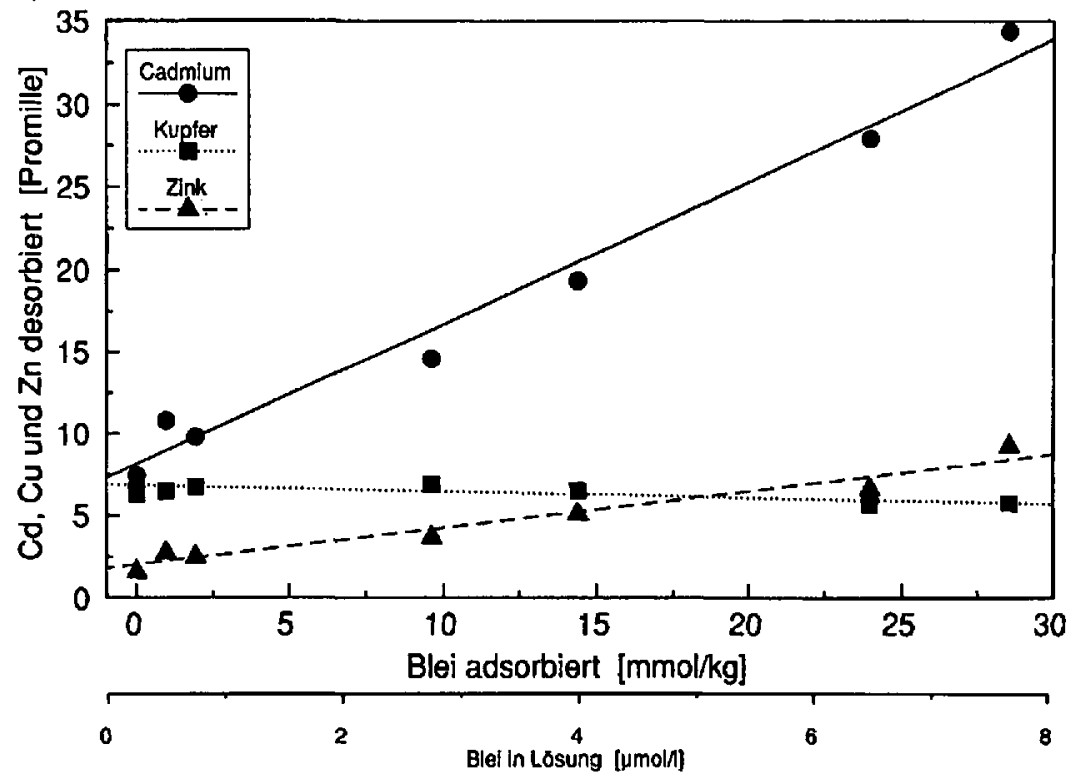

b)

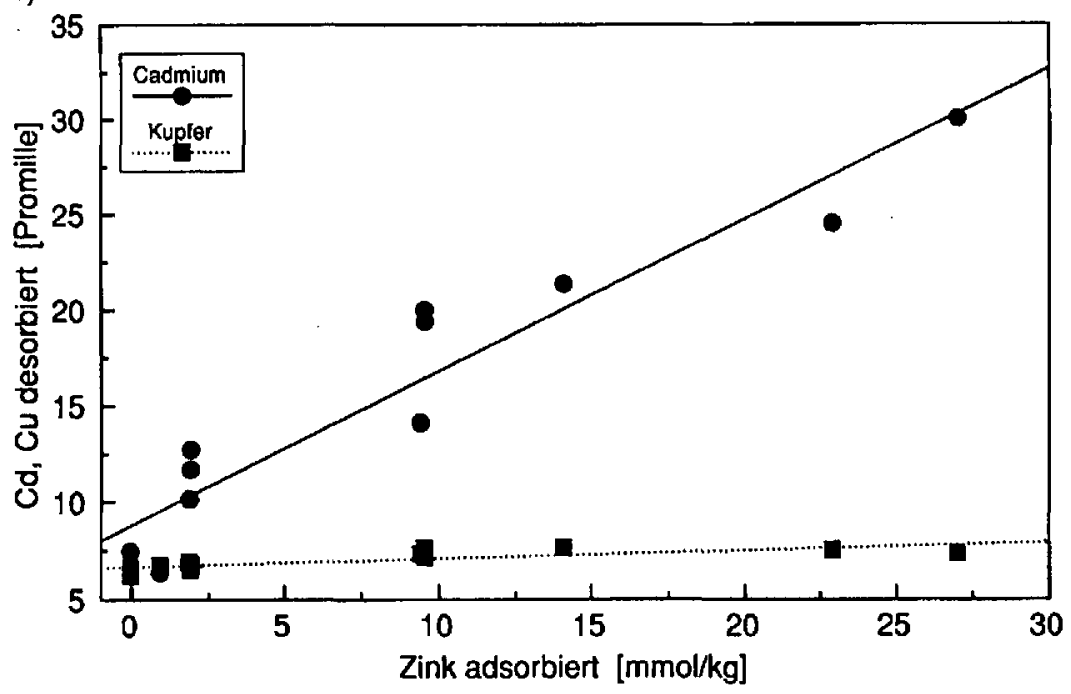

Fig. 6. Desorption von gebundenen Schwermetallen bei der Adsorption von zusätzlichem $P$ bund Zn (Grundelektrolyt:

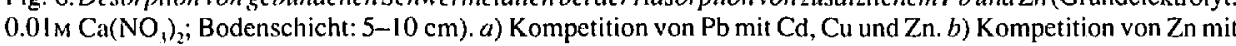
$\mathrm{Cd}$ und $\mathrm{Cu}$.

Bodenlösung mehr als 95\% von $\mathrm{Cu}$ und $\mathrm{Pb}$ an die gelöste organische Substanz gebunden sind, während dieser Anteil bei Cd und $\mathrm{Zn} \mathrm{5 \%} \mathrm{nicht} \mathrm{uibersteigt.} \mathrm{Anderseits} \mathrm{beträgt}$ der Anteil des freien Aqua-Ions bei $\mathrm{Cu}$ lediglich $0.2 \%$ der Gesamtkonzentration in der Lösung, während er bei $\mathrm{Cd}$ und $\mathrm{Zn}$ über $80 \%$ ausmacht. Die tiefe Konzentration des freien $\mathrm{Cu}$ erklärt den geringen $\mathrm{Cu}$-Gehalt in der Vegetation, da Pflanzen Schwermetalle vorwiegend als Aqua-Ionen aufnehmen [25]. Durch Komplexbildungsvorgänge in der Lösungsphase lässt sich auch das ungewöhnliche Verhalten von $\mathrm{Cu}$ bei der Desorption mit Pb-Lösungen erklären. Im Gegensatz zu $\mathrm{Cd}$ und $\mathrm{Zn}$, welche durch Zusatz von steigenden $\mathrm{Pb}$-Mengen zunehmend von der festen Bodenmatrix desorbiert werden (Konkurrenz am Tauscher), erfolgt bei $\mathrm{Cu}$ eher eine Verminderung der Desorption (Fig. 6a). Da die Stabilitätskonstanten für $\mathrm{Pb}-\mathrm{bzw}$. Cu-Humat-Komplexe in der selben Grössenordnung liegen ( $\log K_{2}$ für $\mathrm{Pb}$ :
8.7 [23]), verringert sich infolge der gegenseitigen Konkurrenz um die gelösten Liganden bei steigender $\mathrm{Pb}$-Zugabe der komplexierte Anteil von $\mathrm{Cu}$ in der Lösung. $\mathrm{Zn}$, das schwächer komplexiert wird, verändert die Verteilung von Cu nicht, konkurrenziert aber mit dem noch schwächeren $\mathrm{Cd}$ um die Bindungsplätze (Fig. 6b).

Bei der Sorption an wenig belastetes Bodenmaterial wird pro sorbiertes Äquivalent Schwermetall ein Äquivalent $\mathrm{Ca}$ desorbiert. Der Kationentausch vollzieht sich also hauptsächlich gegen $\mathrm{Ca}$, das Hauptkation an den Tauschern kalkhaltiger Böden. Die Kompetitionsexperimente zeigen, dass auch im stark belasteten Bodenmaterial vorwiegend ein Austausch gegen Ca stattfindet und dass demgegenüber die Konkurrenz der Schwermetalle untereinander gering ist (Tab. 2 und Fig. 6a, b). Trotz starker Schwermetallbelastung ist also noch immer der grösste Teil der Sorptionsstellen mit $\mathrm{Ca}$ belegt, und der Boden ist weit von einer
Schwermetallsättigung entfernt. Ein Durchbrechen der Schwermetallfracht in den Untergrund ist somit nicht zu erwarten.

Die Verfasser danken Frau Linda Birch und den Herren $K$. Barmettler, P. Perret und T. Rüttimann für ihre Unterstützung bei den Analysen und dem Bau der Saugkerzen.

Eingegangen am 14. Juni 1991

[1] D.C. Adriano, 'Trace Elements in the Terrestial Environment', Springer Verlag, New York, 1986.

[2] H. Häni, Chimia 1991, 45. 53.

[3] U. Herms, G. Brümmer, Z.Pflanzenernähr. Bodenk. 1984, 147, 400.

[4] G. Brümmer, K.G.Tiller, U. Herms, P.M.Clayton, Geoderma 1983, 31, 337.

[5] J.F. Mc Carthy, J.M. Zachara, Environ. Sci. Technol. 1989, 23, 496.

[6] U. Gasser, H. Sticher, S. Juchler, Mitt. Disch. Bodenk. Ges. 1989, 59, 339.

[7] Eidg. Departement des Innern, Verordnung über Schadstoffe in Boden (VSBo), 1986.

[8] Kantonales Labor Solothurn, Bericht über Metallgehalte in Boden- und Vegetationsproben aus dem Raum Dornach, 1987.

[9] A. Tessier, P.G.C. Campbell, M. Bisson, Anal. Chem. 1979, 51, 844.

[10] L.M. Shuman, Soil Sci. 1985, 140, 11.

[11] J.P. Singh, S.P.S. Karwasra, Mahendra Singh, Soil Sci. 1988, I46, 359

[12] H. Zeien, G.W. Brümmer, Mitt. Dtsch. Bodenk. Ges. 1989,59, 505.

[13] L. Marquis, 'Schwermetalle und Waldsterben: Untersuchungen im Testgebiet Lägern', Diss. Universität Bern, 1989.

[14] G. Sposito, 'The Chemistry of Soil', Oxford University Press, New York, 1989.

[15] J. Grossmann, 'Physikalische und chemische Prozesse bei der Probennahme von Sickerwasser mittels Saugsonden', Diss. Technische Universität München, 1988.

[16] H. Vogel, A. Desaules, H. Häni, 'Schwermetallgehalte in den Böden der Schweiz., Bericht 40 des Nationalen Forschungsprogrammes «Boden»', Liebefeld-Bern, 1989.

[17] Kantonales Arbeitsinspektorat Solothurn, Staubund Schwermetall-Belastung der Luft in der Umgebung der Metallwerke AG, Dornach, Interner Bericht, 1983.

[18] Gabriella Geiger, 'Keimverhalten und Schwermetallaufnahme von Pflanzen nach verschiedenen Sanierungsmethoden an einem Boden in Dornach', Diplonarbeit am Institut für Terrestrische Oekologie, ETH Zürich, 1990.

[19] G. Tyler, 'Heavy Metals in Soil Biology and Biochemistry, Soil Biochemistry', Eds. E.A. Paul und J.N. Ladd, Marcel Dekker, New York, 1981 , Vol. 5, S. 371

[20] Wei-chun Ma, Environ. Pollut., Ser. A 1984, 33 , 207.

[21] Gabriella Geiger, P.Federer, H. Sticher, 'Einfluss verschiedener Sanierungsmassnahmen auf das Keimverhalten und die Schwermetallaufnahme von Pflanzen auf stark kontaminierten Böden', Bulletin Bodenkundliche Gesellschafr der Scthureiz 1991, 15, im Druck.

[22] D. Saucrbeck, 'Funktion, Güte und Belastbarkeit des Bodens aus agrikulturchemischer Sicht', Kohlhammer, Stultgart, 1985.

[23] F.J.Stevenson, Soil Sci.Soc. Am.J. 1976,40,665.

[24] G. Sposito, J. Coves, SOILCHEM: A Computer Program for the Calculation of Chemical Speciation in Soils, University of California, Riverside, 1988.

[25] F.T. Bringham, J.E. Strong, G. Sposito, Soil Sci. $1983,135,160$.

[26] W.L. Lindsay, 'Chemical Equilibria in Soils', John Wiley \& Sons, New York, 1979. 